\title{
Lipase-catalyzed Remote Kinetic Resolution of Quaternary Carbon-containing Alcohols and Determination of Their Absolute Configuration
}

\author{
Dai Sig Im, Chan Seong Cheong," and So Ha Lee \\ Life Sciences Division, Korea Institute of Science and Technologv. P.O. Box 131. Cheongrvang. Seoul 130-650. Korea \\ Computer-aided Molecular Design \& Bioscience Research Co. Ltd. 464-5. Jung-ri, Kusong-myon. \\ Yongin-shi, Kionggi-do 479-916. Korea \\ Received June 3, 2003
}

\begin{abstract}
The quaternary carbon-containing alcohols (1-6) were resolved enantioselectively by various lipases such as PFL (Pseudomonas fluorescens lipase), LAK (Pseudomonas fluorescens lipase), CRL (Candida rugosa lipase) and PCL (Pseudomonas cepacia lipase). The enzymatic resolution of racemic alcolol $( \pm)$-2 gave the excellent enantioselectivity in favor of $(S)-2 d$ in $99 \%$ ee. while those of the racenic alcohols $(1,3,4,5$ and 6$)$ gave the resolved alcohols with moderate to good enantioselectivity. Also, their absolute configurations were determined by chemical transformation to the known compounds.
\end{abstract}

Key Words : Chiral tertiary nitriles, Lipase. Enantioselective. Resolution

\section{Introduction}

Chiral tertiary benzy'lic nitriles ${ }^{1}$ are valuable building blocks in organic synthesis. as this unique motif can be used to create quaternary chiral centers present in numerous biologically active natural products and pharmaceutical compounds.$^{2-6}$ One of the methods for constnicting of the quaternary stereogenic centers is chemo-enzymatic synthesis. Both whole-cell system and isolated enzymes from these cells are widely used as biocataly st for this purpose. ${ }^{\text {th }}$

In the present report. the efficacies of various lipases from PCL. PFL. LAK and CRL to enantioselectively resolve racemic primary alcohols containing tertiary nitriles (1-6) are presented. Enzymatic enantioselective transesterification of racemic primary alcohols (1-6) using vinyl acetate as an acyl donor was readily accomplished. The absolute configlration of primary alcohols was determined by chemical method.

\section{Experimental Section}

General. ${ }^{\mathrm{l}} \mathrm{H}$ NMR (300 MHz) and ${ }^{13} \mathrm{C}$ NMR spectra $(75$ $\mathrm{MHz}$ ) were recorded on a Varian Gemini $300 \mathrm{MHz}$ spectrometer with TMS as an internal reference. Optical rotation was measured on Autopol ${ }^{(5)}$ III polarimeter (Rudolph Research Co.). Low EI resolution mass spectra were determined on HP GC 5972 (column: Hewlett-Packard fused silica capillary column HP-5 cross-linked $5 \%$ phenyl methyl silicone. column ID $0.20 \mathrm{~mm}$. film thickness: 0.11 $\mu \mathrm{m}$. length: $25 \mathrm{~m}$. detector : mass selective detector. $280^{\circ} \mathrm{C}$. injector $280^{\circ} \mathrm{C}$. program: initial temp $70^{\circ} \mathrm{C}(2 \mathrm{~min}) .20^{\circ} \mathrm{C} /$ min. final temp. $300^{\circ} \mathrm{C}$ ) and HP MS $5988 \mathrm{~A}$ sy stem at $70 \mathrm{eV}$. Analytical HPLC works were carried out on Varian 9010 solvent delivery sy'stem. Varian 9050 variable wavelength UV-Vis detector and Varian 4400 integrator using the chiral columns such as Chiralcel OD. Chiralcel OJ. Chiralpak $\mathrm{AD}$ and $\mathrm{OB}(250 \times 4.6 \mathrm{~mm}$. Daicel) for substrate alcohols $(\mathbf{1 - 6})$
Materials. Column chromatography was performed on Merck Silica Gel 60 (230-400 mesh). TLC was carried out using glass sheets precoated with silica gel $60 \mathrm{~F}_{5 \leqslant 4}$ prepared by E. Merck. All the commercially available reagents were obtained from Aldrich. Fluka and Tokyo Kasei Chemical and generally used without further purification. Solvents were distilled over appropriate drying materials before use. PCL (lipase from Pseudomonas cepacia lipase. $30.000 \mathrm{u} / \mathrm{g}$ ) and Lipase AK (Pseudomonas fluorescens lipase. $>20,000$ $\mathrm{u} / \mathrm{g})$ were purchased from Amano enzyme Co. Ltd. CRL (Candida rugosa lipase. $860 \mathrm{w} / \mathrm{mg}$ ) and PFL (Pseudomonas fluorescens lipase. $42.5 \mathrm{u} / \mathrm{mg}$ ) were purchased from Sigma and Aldrich. respectively

(士)-4-Cyano-4-(4-nitrophenyl)-1-hexanol (1) and its derivatives

(土)-2-(4-Nitrophenyl)-5-(tetrahydropyran-2-yloxy)pentanenitrile (1b). To a stirred suspension of $60 \%$ sodium hydride ( $1.92 \mathrm{~g} .48 .0 \mathrm{mmol})$ in dry DMF (70 mL) was added dropwise 4-nitropheny lacetonitrile (1a) $(6.62 \mathrm{~g} .40 .0 \mathrm{mmol})$ for $30 \mathrm{~min}$ at $0{ }^{\circ} \mathrm{C}$. After the mixture was stirred for $30 \mathrm{~min}$ 3-tetrahydropy ranyloxy propyl bromide ( $10.0 \mathrm{~g} .45 .0 \mathrm{mmol}$ ) was added and the mixtures were further stirred at $15^{\circ} \mathrm{C}$ for $12 \mathrm{~h}$. The reaction medium was quenched with cold ice water $(50 \mathrm{~mL})$ and extracted with diethyl ether $(2 \times 70 \mathrm{~mL})$. The organic extracts were washed with saturated aqueous $\mathrm{NaHCO}_{3}$ solution, brine, dried over anhy'drous $\mathrm{MgSO}_{4}$ and concentrated to furnish the cyanide $1 \mathrm{~b}$. The cyanide $\mathbf{1 b}$ was purified by column chromatography ( $n$-hexane/EtOAc $=$ $10 / 1, \mathrm{v} / \mathrm{v})$.

Yield ( $9.7 \mathrm{~g}$ ) $80 \%$ : Rf 0.48 (benzene/EtOAc. $5 / 1$. v/v): GC/ MSD retention time (min) $13.01, \mathrm{~m} / \mathrm{z} 56,67.77 .85$ (100). 101, 115, 130, 142, 157, 174, 185, 204, 218, 231, 246. 259. 269. 277. 287. $304\left(\mathrm{M}^{+}\right):{ }^{1} \mathrm{H}$ NMR $\left(300 \mathrm{MHz}, \mathrm{CDCl}_{3}\right) \delta$ $1.43-1.49(\mathrm{~m} .4 \mathrm{H}) .1 .55-2.00(\mathrm{~m} .5 \mathrm{H}), 2.37-2.45(\mathrm{~m} .2 \mathrm{H})$. $3.39-3.44(\mathrm{~m}, 2 \mathrm{H}), 3.72-3.77$ (m. $2 \mathrm{H}), 4.50$ (t. $J=3.4 \mathrm{~Hz}$. IH). $8.05($ d. $J=8.9 \mathrm{~Hz}, 2 \mathrm{H}) .8 .22(\mathrm{~d} . J=8.9 \mathrm{~Hz}, 2 \mathrm{H}):{ }^{13} \mathrm{C}$ NMR (75 MHz, $\left.\mathrm{CDCl}_{3}\right) \delta 14.5,20.0 .25 .6 .31 .0,36.2,62.3$. 
$65.2,99.2 .119 .9,124.0,139.4,1+1.8,150.5$

(土)-2-Ethyl-2-(4-nitrophenyl)-5-(tetrahydropyran-2yloxy)pentanenitrile (1c). Compound 1c was sytnthesized by the same method as compound 1b using compound 1b $(4.0 \mathrm{~g} .13 .1 \mathrm{mmol})$ and iodoethane $(1.26 \mathrm{~mL}, 15.8 \mathrm{mmol})$.

Yield (4.15 g) 95\%: $\mathrm{R}_{\mathrm{f}} 0.33$ (n-hexane/EtOAc. 2/1, v/v): GC/MSD retention time (min) $13.23 . \mathrm{m} / \mathrm{z} 56,67.76 .85$ (100), 101, 115, 128, 143, 155, 170, 189, 204, 214, 231, 260. $274,287,305,315,332\left(\mathrm{M}^{+}\right):{ }^{\mathrm{l}} \mathrm{H} \mathrm{NMR}\left(300 \mathrm{MHz}, \mathrm{CDCl}_{3}\right) \delta$ $0.88(\mathrm{t} . J=7.3 \mathrm{~Hz}, 3 \mathrm{H}), 1.30-1.5 \mathrm{l}(\mathrm{m}, 5 \mathrm{H}), 1.60-1.81(\mathrm{~m}$. $3 \mathrm{H}) .1 .91-2.50(\mathrm{~m} .5 \mathrm{H}), 3.33-3.46(\mathrm{~m} .2 \mathrm{H}), 3.65-3.79(\mathrm{~m}$. $2 \mathrm{H}) .4 .43-4.50(\mathrm{~m} . \mathrm{HH}) .7 .57$ (d. $J=8.9 \mathrm{~Hz}, 2 \mathrm{H}) .8 .23$ (d. $J=$ $8.9 \mathrm{~Hz}, 2 \mathrm{H}):{ }^{13} \mathrm{C} \mathrm{NMR}\left(75 \mathrm{MHz}, \mathrm{CDCl}_{3}\right) \delta 9.9 .19 .1 .25 .7$. $26.1,30.9,34.4,37.5,49.4 .62 .8,66.7,99.2,121.5,124.4$. 127.6. 146.0.147.7.

(t)-4-Cyano-4-(4-nitrophenyl)-1-hexanol (1). To a solution of compound 1c (3.2 g. $9.63 \mathrm{mmol})$ in methanol (40 $\mathrm{mL})$ was added $\mathrm{IN}$ methanolic $\mathrm{HCl}(10 \mathrm{~mL})$, and stirred for $10 \mathrm{~h}$ at $25^{\circ} \mathrm{C}$. The reaction medium was quenched with cold ice water $(50 \mathrm{~mL})$ and extracted with diethyl ether $(100 \mathrm{~mL})$. The organic extracts were washed with saturated aqueous $\mathrm{NaHCO}_{3}$ solution. brine. dried over anhydrous $\mathrm{MgSO}_{4}$ and concentrated to furnish the cyranide 1. Compound 1 was purified by column chromatography ( $n$-hexane/EtOAc $=4 / 1$. v/v).

Yield $\left(2.1\right.$ g) $88 \%$ : mp 94-97 $\mathrm{C}: \mathrm{R}_{\mathrm{f}} 0.10$ (n-hexane/ EtOAc. 2/1, v/v): GC/MSD retention time (min) $11.88, \mathrm{~m} / \mathrm{z}$ $51,63,77,89,102,128,140,155,175,190(100), 201,218$. 230. $248\left(\mathrm{M}^{+}\right):{ }^{1} \mathrm{H}$ NMR $\left(300 \mathrm{MHz}, \mathrm{CDCl}_{3}\right) \delta 0.90$ (t. $J=$ $7.3 \mathrm{~Hz}, 3 \mathrm{H}), 1.23-1.37(\mathrm{~m}, \mathrm{lH}), 1.62-\mathrm{I} .78(\mathrm{~m}, \mathrm{lH}), 1.92-$ $2.14(\mathrm{~m} .4 \mathrm{H}) .2 .24(\mathrm{~s} .1 \mathrm{H}) .3 .57(\mathrm{t} . J=6.1 \mathrm{~Hz} .2 \mathrm{H}) .7 .64(\mathrm{~d} . J$ $=9.0 \mathrm{~Hz}, 2 \mathrm{H}) .8 .20(\mathrm{~d} . J=9.0 \mathrm{~Hz} \cdot 2 \mathrm{H}):{ }^{13} \mathrm{C} \mathrm{NMR}(75 \mathrm{MHz}$. $\left.\mathrm{CDCl}_{3}\right) \delta 9.9,28.7,34.4,37.2,49.4 .62 .0,121.6,124.5$. 127.6. 141.0. 147.8: Anal. Calcd for $\mathrm{C}_{13} \mathrm{H}_{10} \mathrm{~N}_{2} \mathrm{O}_{3}$ : C. 62.89 : H. $6.50:$ N. 11.28. Found: C. 62.6: H. 6.57: N. 10.8: HPLC analỳsis (Chiralcel OD column $n$-hexane/IPA, 8/2, v/v). retention time (min) $10.18(S)$ and $11.7+(R)$.

( \pm -4-Cyano-4-(4-nitrophenyl)hexyl acetate (1d). To a solution of $( \pm)-1(1.0 \mathrm{~g} .4 .0 \mathrm{mmol})$ was added 4-(dimethỵlamino)py ridine $(10 \mathrm{mg}) . \mathrm{Et}_{3} \mathrm{~N}(1.68 \mathrm{~mL}, 12.0 \mathrm{mmol})$ and acetic anhydride (1.1 mL, $8.0 \mathrm{mmol})$ in $\mathrm{CH}_{2} \mathrm{Cl}_{2}(30 \mathrm{~mL})$. The mixture was stirred at $25{ }^{\circ} \mathrm{C}$ for $5 \mathrm{~h}$. The reaction mixture was neutralized with $2 \%$ aqueous $\mathrm{HCl}$ solution and extracted with $\mathrm{CH}_{2} \mathrm{Cl}_{2}(50 \mathrm{~mL})$. The organic layer was washed with saturated aqueous $\mathrm{NaHCO}_{3}$ solution. brine. dried over anlyydrous $\mathrm{MgSO}_{4}$ and concentrated to afford ( \pm )1d which was purified by column chromatography ( $n$ hexane/ethyl acetate. 10/1,v/v).

Yield $\left(1.0\right.$ g) $86 \%: \mathrm{R}_{\mathrm{f}} 0.3+(n$-hexane/EtOAc. $2 / 1, \mathrm{v} / \mathrm{v})$ : GC/MSD retention time (min) $11.86,(\mathrm{~m} / \mathrm{z}) 50.63 .73 .89$. $101(100), 115,128,1+3,155,175,190,202,212,221,230$. $248,273,290\left(\mathrm{M}^{+}\right):{ }^{\mathrm{l}} \mathrm{H}$ NMR $\left(300 \mathrm{MHz}, \mathrm{CDCl}_{j}\right) \delta 0.91(\mathrm{t} . J$ $=7 .+\mathrm{Hz} .3 \mathrm{H}) .1 .38-1.4+(\mathrm{m} . \mathrm{HH}) .1 .80-1.85(\mathrm{~m} .1 \mathrm{H}) .1 .95-$ $2.18(\mathrm{~m} .4 \mathrm{H}) .2 .04(\mathrm{~s} .3 \mathrm{H}) .4 .03(\mathrm{t} . J=6.2 \mathrm{~Hz} .2 \mathrm{H}) .7 .62(\mathrm{~d} . J$ $=11.3 \mathrm{~Hz}, 2 \mathrm{H}) \cdot 8.30(\mathrm{~d}, J=11.3 \mathrm{~Hz}, 2 \mathrm{H}):{ }^{13} \mathrm{C} \operatorname{NMR}(75$ $\left.\mathrm{MHz}, \mathrm{CDCl}_{3}\right) \delta 21.2 .25 .1,34.6 .37 .5,49.4,63.6 .121 .3$. 124.6. 127.5, 145.5. 171.2: HPLC analỵsis (Chiralcel OD column, n-hexane/IPA. 8/2, v/v), retention time (min) 13.63 $(S)$ and $15.57(R)$.

( \pm -4-Cyano-4-phenyl-1-octanol (3) and its derivatives (t)-4-Cyano-4-phenyl-1-octanol (3). Compound 3 was synthesized by the same method as compound 1 using comound $3 \mathrm{c}$ ( $3.8 \mathrm{~g} .12 .0 \mathrm{mmol})$.

Yield (1.78 g) 62\%: $\mathrm{R}_{\mathrm{f}} 0.38$ (n-hexane/EtOAc. $2 / \mathrm{l}$. v/v): GC/MSD retention time (min) 9.62. (m/z) 51, 57, 77, 91. 103, 116, $129(100), 142,156,172,189,204,231\left(\mathrm{M}^{+}\right):{ }^{1} \mathrm{H}$ NMR $\left(300 \mathrm{MHz}, \mathrm{CDCl}_{3}\right) \delta 0.84(\mathrm{t}, J=7.3 \mathrm{~Hz}, 3 \mathrm{H}), 1.03-$ $1.20(\mathrm{~m}, \mathrm{IH}) .1 .22-1.48(\mathrm{~m}, 4 \mathrm{H}) .1 .50(\mathrm{~s} . \mathrm{HH}) .1 .63-1.78(\mathrm{~m}$. IH). $1.84-2.17(\mathrm{~m}, 4 \mathrm{H}), 3.59(\mathrm{t} . J=6.2 \mathrm{~Hz}, 2 \mathrm{H}), 7.30-7.40$ (m, 5H): Anal. Calcd for $\mathrm{C}_{1 \leqslant} \mathrm{H}_{21} \mathrm{NO}: \mathrm{C} .77 .88: \mathrm{H}, 9.15: \mathrm{N}$, 6.05. Found: C. 77.1: H. 9.18: N. 5.94: HPLC analysis (Chiralcel OD, n-hexane/IPA, 95/5, v/v), retention time (min). $29.22(R)$ and $31.25(S)$ : HPLC analysis (Chiralcel OJ. n-hexane/IPA. 94/6. v/v). retention time (min). $16.48(S)$ and $21.12(R)$ : $(R)-(-)-3\left(>99 \%\right.$ ee) $[\alpha]_{\mathrm{D}}^{23}-8.34(c 0.20$. $\mathrm{MeOH})(S)-(+)-3(>99 \%$ ee $)[\alpha]_{\mathrm{D}}^{\hat{2}}+8.33(c 0.20 . \mathrm{MeOH})$.

( \pm -4-Cyano-4-phenyloctyl acetate (3d). Compound 3d was synthesized by the same method as compound 1d using compound $3(0.7 \mathrm{~g} .3 .0 \mathrm{mmol})$.

Yield $(0.79 \mathrm{~g}) 96 \%: \mathrm{R}_{\mathrm{f}} 0.78$ (n-hexane/EtOAc. $\left.2 / \mathrm{l}, \mathrm{v} / \mathrm{v}\right)$ : GC/MSD retention time (min) $10.51, \mathrm{~m} / 2 \mathrm{z} 1,77,10 \mathrm{l}, 116$, $129(100), 145,156,175,187,213,230,246,273\left(\mathrm{M}^{+}\right):{ }^{1} \mathrm{H}$ NMR $\left(300 \mathrm{MHz}, \mathrm{CDCl}_{3}\right) \delta 0.83$ (t. $\left.J=7.24 \mathrm{~Hz}, 3 \mathrm{H}\right) .0 .95-$ $1.18(\mathrm{~m}, \mathrm{lH}) .1 .25-1.32(\mathrm{~m}, 2 \mathrm{H}), 1.39-1.43(\mathrm{~m} .2 \mathrm{H}) .1 .75-$ $2.10(\mathrm{~m}, 5 \mathrm{H}), 2.05$ (s. 3H). $4.00(\mathrm{t} . J=6.19 \mathrm{~Hz}, 2 \mathrm{H}), 7.28-$ $7.38(\mathrm{~m}, 5 \mathrm{H}):{ }^{13} \mathrm{C} \mathrm{NMR}\left(75 \mathrm{MHz}, \mathrm{CDCl}_{3}\right) \delta 14.1,21.2,22.8$. $25.0 .27 .6,37.9,41.2,47.9 .63 .9,122.2,127.7,129.5,134.1$, 137.1. 171.3: HPLC analysis (Chiralcel OD, n-hexane/IPA. 95/5, v/v). retention time (min). $14.41(S)$ and $19.15(R)$ : (S)$(+)-3 d\left(>56 \%\right.$ ee) $[\alpha]_{\mathrm{D}}^{23}+4.1(c 1.0 . \mathrm{MeOH})$.

$( \pm)-4-(+-C h l o m p h e n y l)-4-c y a n o-1-o c t a n o l ~(4)$ and its derivatives

(t)-4-(4-Chlorophenyl)-4-cyano-1-octanol (4). Compound 4 was synthesized by the same method as compound 1 using compound $\mathbf{4 c}$

Yield $(0.3 \mathrm{~g}) 79 \%: \mathrm{R}_{\mathrm{f}} 0.38$ (n-hexane/EtOAc. 2/1. v/v): GC/MSD retention time (min) 10.83, (m/z) 57.77.85.101 115, 128, 150163 (100), 179, 190, 206, 238,247, $265\left(\mathrm{M}^{+}\right)$: ${ }^{\mathrm{l}} \mathrm{H}$ NMR $\left(300 \mathrm{MHz}, \mathrm{CDCl}_{3}\right) \delta 0.83(\mathrm{t} . J=7.2 \mathrm{~Hz}, 3 \mathrm{H}), 1.03-$ $1.20(\mathrm{~m}, \mathrm{lH}) .1 .25-1.35(\mathrm{~m}, 4 \mathrm{H}), 1.40-1.48(\mathrm{~m} . \mathrm{lH}) .1 .52-$ $1.89(\mathrm{~m},+\mathrm{H}) .2 .1 \mathrm{l}(\mathrm{s} . \mathrm{HH}) .3 .55(\mathrm{t} . J=6.0 \mathrm{~Hz} .2 \mathrm{H}) .7 .30-$ $7.37(\mathrm{~m}, 5 \mathrm{H}):{ }^{13} \mathrm{C} \mathrm{NMR}\left(75 \mathrm{MHz}, \mathrm{CDCl}_{3}\right) \delta 14.1,22.8,27.7$. 28.7, 37.7, 41.2, 48.0, 62.2, 122.5, 127.7, 129.4, 133.9 , 137.4: Anal. Calcd for $\mathrm{C}_{15} \mathrm{H}_{21} \mathrm{ClNO}: \mathrm{C}, 67.79: \mathrm{H}, 7.58: \mathrm{N}$. 5.27. Found: C. 67.8: H, 7.73: N. 5.29: HPLC analysis (Chiralcel OD, $n$-hexane/IPA, 95/5, v/v), retention time (min). $39.58(S)$ and $44.18(R)$.

$( \pm)-4-(+-C h l o r o p h e n y l)-4-c y a n o o c t y l$ acetate (4d). Compound td was synthesized by the same method as compound 1d using compound $+(0.8 \mathrm{~g} .3 .0 \mathrm{mmol})$.

Yield $(0.83 \mathrm{~g}) 90 \%: \mathrm{R}_{\mathrm{f}} 0.78$ (n-hexane/EtOAc. $2 / \mathrm{l}$. v/v): ${ }^{\mathrm{l}} \mathrm{H} \mathrm{NMR}\left(300 \mathrm{MHz}, \mathrm{CDCl}_{3}\right) \delta 0.83(\mathrm{t} . J=7.2 \mathrm{~Hz}, 3 \mathrm{H}), 0.95-$ $1.18(\mathrm{~m}, \mathrm{lH}) .1 .25-1.32(\mathrm{~m}, 2 \mathrm{H}), 1.39-1.43(\mathrm{~m} .2 \mathrm{H}) .1 .75-$ $2.10(\mathrm{~m}, 5 \mathrm{H}), 2.05$ (s. $3 \mathrm{H}) .+.00(\mathrm{t} . J=6.19 \mathrm{~Hz} .2 \mathrm{H}) .7 .28-$ 
$7.38(\mathrm{~m}, 4 \mathrm{H}):{ }^{13} \mathrm{C} \mathrm{NMR}\left(75 \mathrm{MHz}, \mathrm{CDCl}_{3}\right) \delta 14.1,21.2 .22 .8$. 25.0 .27 .6 .37 .9 .41 .2 .47 .9 .63 .9 .122 .2 . 127.7. 129.5. 134.1. 137.1. 171.3: HPLC analy sis (Chiralcel OD. $n$-hexane/IPA. 95/5. v/v) retention time (min). $14.41(S)$ and $19.15(R)$.

( \pm -4-Cyano-4-cyclohexyl-4-phenyl-1-butanol (5) and its derivatives

( \pm )-2-Phenyl-5-(tetrahydro-pyran-2-yloxy)pentanenitrile (5b). To a stirred suspension of $60 \%$ sodium hydride $(1.2 \mathrm{~g}$. $29.0 \mathrm{mmol}$ ) in dry DMF $(50 \mathrm{~mL})$ was added dropwise benzy'l cyanide $(3.1 \mathrm{~mL} .26 .4 \mathrm{mmol})$ for $30 \mathrm{~min}$ at $0{ }^{\circ} \mathrm{C}$. After the mixture was stirred for $30 \mathrm{~min}$. 3-tetrahydropy rany loxypropyl bromide $(5.89 \mathrm{~g} .26 .4 \mathrm{mmol})$ was added for $30 \mathrm{~min}$ and the mixtures were further stirred at $15^{\circ} \mathrm{C}$ for $12 \mathrm{~h}$. The reaction medium was quenched with cold ice water $(50 \mathrm{~mL})$ and extracted with diethyl ether $(2 \times 50 \mathrm{~mL})$. The organic extracts were washed with saturated aqueous $\mathrm{NaHCO}_{3}$ solution. brine. dried over anhydrous $\mathrm{MgSO}_{4}$ and concentrated to furnish the cyanide $\mathbf{5 b}$. The compound $\mathbf{5 b}$ was purified by column chromatography (n-hexane/ethyl acetate $=4 / 1, \mathrm{v} / \mathrm{v})$

Yield $(5.82 \mathrm{~g}) 85 \%: \mathrm{R}_{\mathrm{f}} 0.52$ (n-hexane/EtOAc. $2 / 1$, v/v): GC/MSD retention time (min) $13.32 . \mathrm{m} / \mathrm{z} 55,67.85(100)$. 101. 129.143, 156. 189. 198, 216,234. $259\left(\mathrm{M}^{+}\right):{ }^{\mathrm{H}} \mathrm{H}$ NMR $\left(300 \mathrm{MHz}, \mathrm{CDCl}_{3}\right) \delta 1.48-1.53(\mathrm{~m}, 4 \mathrm{H}) .1 .56-1.92(\mathrm{~m}, 4 \mathrm{H})$. $1.98-2.08(\mathrm{~m} .2 \mathrm{H}) .3 .38-3.56(\mathrm{~m} .2 \mathrm{H}) .3 .75-3.83(\mathrm{~m} .2 \mathrm{H})$. $3.87(\mathrm{t} . J=7.4 \mathrm{~Hz}, \mathrm{lH}), 4.52-4.57(\mathrm{~m} . \mathrm{lH}) .7 .28-7.40(\mathrm{~m}$. $5 \mathrm{H}):{ }^{13} \mathrm{C} \mathrm{NMR}\left(75 \mathrm{MHz}, \mathrm{CDCl}_{3}\right) \delta 20.0,25.8,27.5 .31 .0$. 33.4, 37.5. 62.8, 66.8, 99.3, 121.2, 127.7, 128.4. 129.4. 136.2 .

(土)-2-Cyclohexyl-2-phenyl-5-(tetrahydropyran-2-yloxy)pentanenitrile $(5 \mathrm{c})$. The reaction was progressed by the same method as compound 1c using compound $5 \mathrm{~b}$ ( $2.6 \mathrm{~g}$. $10.02 \mathrm{mmol}$ ) and bromocyclohexane $(1.23 \mathrm{~mL}, 10.02 \mathrm{mmol})$.

Yield $\left(3.0\right.$ g) $82 \%$ : $\mathrm{R}_{\mathrm{f}} 0.49$ ( $n$-hexane/EtOAc. $4 / 1$, v/v): GC/MSD retention time (min) $13.60 \mathrm{~m} / \mathrm{z} 55.85(100), 101$.

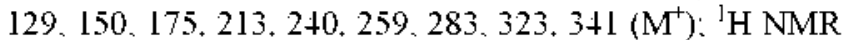
$\left(300 \mathrm{MHz}, \mathrm{CDCl}_{3}\right) \delta 1.06-1.12(\mathrm{~m}, 2 \mathrm{H}) .1 .20-1.30(\mathrm{~m} .+\mathrm{H})$. $1.50-2.03(\mathrm{~m} .13 \mathrm{H}) .2 .05-2.11(\mathrm{~m} . \mathrm{lH}) .2 .15-2.30(\mathrm{~m} . \mathrm{lH})$. 3.29-3.35 (m. IH). 3.45-3.49 (m. IH). 3.63-3.67 (m. IH). $3.74-3.78(\mathrm{~m}, \mathrm{lH}) .4 .45-4.51(\mathrm{~m}, \mathrm{lH}) .7 .27-7.37(\mathrm{~m}, 5 \mathrm{H})$ : ${ }^{13} \mathrm{C}$ NMR $\left(75 \mathrm{MHz} . \mathrm{CDCl}_{3}\right) \delta 20.0 .26 .1,26.3 .26 .7 .28 .9$. $29.1,31.0,34.3,47.4,53.3,62.7,62.8,67.2,99.1,122.0$. 126.9. 127.8,129.0,138.3

( \pm )-4-Cyano-4-cyclohexyl-4-phenyl-1-butanol (5). Compound $\mathbf{5}$ was synthesized by the same method as compound 1 using compound $\mathbf{5 e}$ ( $3 \mathrm{~g} .8 .78 \mathrm{mmol}$ ).

Yield $(2.1 \mathrm{~g})$ 91\%: GC/MSD retention time (min) 11.76. $\mathrm{m} / \mathrm{z} 55,73,89,103,115,129(100), 1+2,158,175,221,237$. $257(\mathrm{M}):{ }^{1} \mathrm{H}$ NMR $\left(300 \mathrm{MHz}, \mathrm{CDCl}_{3}\right) \delta 1.05-1.27$ (m. 6H). $1.56-1.73(\mathrm{~m} .+\mathrm{H}) .1 .83-2.25$ (m. $+\mathrm{H}) .2 .03$ (s. $1 \mathrm{H}) .3 .46-$ $3.55(\mathrm{~m} .2 \mathrm{H}), 4.07-4.12(\mathrm{~m} . \mathrm{lH}), 7.28-7.37(\mathrm{~m} .5 \mathrm{H}):{ }^{13} \mathrm{C}$ NMR $\left(75 \mathrm{MHz}, \mathrm{CDCl}_{3}\right) \delta 14.5,21.4,26.3,28.9,29.1 .34 .0$. 47.5. 53.3. 60.8. 62.5. 122.1. 126.8. 129.1 138.1: Anal. Calcd for $\mathrm{C}_{17} \mathrm{H}_{23} \mathrm{NO}:$ C. $79.33:$ H. 9.01: N. 5.44. Found: $\mathrm{C}$. 79.4: H. 8.98: N. 5.41: HPLC analysis (Chiralcel OD. $n$ hexane/iso-PrOH. 95/5, v/v), retention time (min). $50.64(S)$ and $57.39(R):(R)$ form $[\alpha]_{\mathrm{D}}^{3+4}+11.4(\mathrm{c}=0.25 . \mathrm{MeOH}) 99 \%$ ee.
(士)-4-Cyano-4-cyclohexyl-4-phenylbutyl acetate (5d). Compound $5 \mathbf{d}$ was synthesized by the same method as compound 1d using compound $5(0.8 \mathrm{~g} .3 .1 \mathrm{mmol})$.

Yield $(0.85 \mathrm{~g}) 91 \%$ : ${ }^{1} \mathrm{H}$ NMR $\left(300 \mathrm{MHz}, \mathrm{CDCl}_{3}\right) \delta 1.06-$ $1.28(\mathrm{~m}, 6 \mathrm{H}) .1 .53-1.76(\mathrm{~m}, 4 \mathrm{H}), 1.83-2.25(\mathrm{~m}, 4 \mathrm{H}), 2.36(\mathrm{~s}$. $3 \mathrm{H})$. 3.44-3.56 (m. 2H). 4.06-4.11 (m, lH). 7.29-7.38 (m. $5 \mathrm{H}):{ }^{13} \mathrm{C}$ NMR $\left(75 \mathrm{MHz}, \mathrm{CDCl}_{3}\right) \delta 13.8 .21 .7,26.8,28.8$. $29.3,34.4,47.5,53.3,60.8,62.5,122.1,126.8,129.1,138.1$, 172.3: HPLC analysis (Chiralcel OD. $n$-hexane/iso-PrOH, 95/5, v/N) retention time (min). $10.73(S)$ and $13.91(R)$ : $(S)$ form $[\alpha]_{D}^{-+}-15.1(\mathrm{c}=1.8 \mathrm{MeOH}) 99 \%$ ee

$( \pm)-5$-Cyano-5-phenyl-1-nonanol (6) and its derivatives

( \pm )-5-Cyano-5-phenyl-1-nonanol (6). Compound 6 was synthesized by the same method as compound 1 using compound $6 \mathrm{c}(6 \mathrm{c})(2.90 \mathrm{~g} .8 .8 \mathrm{mmol})$.

Yield $(1.81$ g) $83 \%$ : Rf 0.29 (n-hexane/EtOAc. 1/L. v/v): GC/MSD retention time (min) $10.42 . \mathrm{m} / \mathrm{z} 55,65.77,91$. $103,116,129,143,158,173(100), 189,202,218,227,245$ (M): ${ }^{l} \mathrm{H} \mathrm{NMR}\left(300 \mathrm{MHz}, \mathrm{CDCl}_{3}\right) \delta 0.84(\mathrm{t} . J=7.3 \mathrm{~Hz}, 3 \mathrm{H})$. $1.0 \mathrm{l}-\mathrm{I} .38(\mathrm{~m} .5 \mathrm{H}) .1 .39-1.60(\mathrm{~m} .3 \mathrm{H}) .1 .50(\mathrm{~s} . \mathrm{HH}) .1 .81-$ $2.09(\mathrm{~m}, 4 \mathrm{H}) .3 .58(\mathrm{t} . J=6.2 \mathrm{~Hz}, 2 \mathrm{H}), 7.27-7.39(\mathrm{~m}, 5 \mathrm{H})$ : HPLC analysis (Chiralpak AD, $n$-hexane/PA. 94/6. v/v). retention time (min). $31.34(R)$ and $36.78(S)$.

( \pm )-5-Cyano-5-phenylnonyl acetate (6d). Compound 6d was synthesized by the same method as compound $1 \mathbf{d}$ using compound $6(0.4 \mathrm{~g} .1 .64 \mathrm{mmol})$.

Yield $(0.4 \mathrm{~g}) 88 \%$ : Rf 0.52 ( $n$-hexane/EtOAc, I/l, v/v): GC/MSD retention time (min) 10.90. (m/z) 55.61. 91. 103, 115. 123 (100), 145. 173. 189. 200, 227, 244, 260. $287\left(\mathrm{M}^{+}\right)$: HPLC analysis (Chiralpak AD, $n$-hexane/IPA, 94/6, v/v). retention time (min), 8.50 (S) and $9.40(R)$.

General procedure for the enzymatic kinetic transesterification of racemic alcohols (1-6) using several lipases. To a stirred solution of the primary racemic alcohols (1-6) (1.0 mmol) in anlydrous $n$-hexane or the mixed solvent of $n$ hexane and ethyl acetate was added any lipase in PCL (half mass). PFL ( $10 \%$ mass). LAK (half mass). and CRL (equivalent mass) and vinyl acetate ( $89 \mathrm{mg} .1 .0 \mathrm{mmol}$ ) as an acyl donor at $32-3+{ }^{\circ} \mathrm{C}$ and the progress of the reaction was monitored by chiral column of HPLC. The reaction mixture was diluted with diethyl ether and the enzy'me was removed by filtration and the organic solvent was evaporated under reduced pressure. The reaction residue was chromatographed on silica-gel column with the mixed solvent of $n$ hexane and ethyl acetate to give the reacted acetates of each alcohol and unreacted alcohol. The isolated acetate was hydrolyzed with $1 \mathrm{~N}$ methanolic $\mathrm{KOH}$ solution to afford the corresponding alcohol.

Syntheses of the compounds for the determination of absolute configuration of the resolved alcohol $(R)-3$

(S)-2-Formyl-2-phenylhexanenitrile ((S)-8). To a solution of (S)-2-cyano-2-phenyl-1-hexanol (7) $(0.47 \mathrm{~g} .2 .32 \mathrm{mmol})$ in methylene chloride $(20 \mathrm{~mL})$ was added PCC $(0.6 \mathrm{~g} .2 .78$ mmol) and stirred at $\mathrm{rt}$ for $5 \mathrm{~h}$. The reaction mixture was concentrated and chromatography ( $n$-hexane/EtOAc. $4 / 1$, $\mathrm{v} / \mathrm{v})$ of the residue gave the pure $(S)-8$.

Yield $(0.4 \mathrm{~g}) 86 \%: \mathrm{R}_{i} 0.52$ ( $n$-hexane/EtOAc. $\left.4 / 1, \mathrm{v} / \mathrm{v}\right)$ : 
GC/MSD retention time (min) 7.69. (m/z) 51.63.77.89. 103 (100). 117, 130, 145, 158, 173, 201245 (M): ${ }^{1} \mathrm{H}$ NMR $\left(300 \mathrm{MHz}, \mathrm{CDCl}_{3}\right) \delta 0.87(\mathrm{t} . J=7.3 \mathrm{~Hz} .3 \mathrm{H}) .1 .18-1.45(\mathrm{~m}$. $4 \mathrm{H}) .1 .87-2.11(\mathrm{~m} .2 \mathrm{H}) .7 .35-7.47(\mathrm{~m} .5 \mathrm{H}) .8 .03$ (s. $1 \mathrm{H}$ ).

trans $(R)$-4-Cyano-4-phenyloct-2-enoic acid methyl ester $((\boldsymbol{R})-9)$. To a solution of $(R)-8(0.12 \mathrm{~g} .0 .6 \mathrm{mmol})$ in methylene chloride ( $15 \mathrm{~mL}$ ) was added methyl (tripheny'lphosphoranylidene)acetate $(0.23 \mathrm{~g} .0 .70 \mathrm{mmol})$ and stirred at $\mathrm{rt}$ for $2 \mathrm{~h}$. The mixture was concentrated and chromatography (nhexane/EtOAc. 10/1, v/v) of the residue gave the pure $(R)-9$.

Yield $(0.13 \mathrm{~g}) 85 \%: \mathrm{R}_{\mathrm{f}} 0.37$ (n-hexane/EtOAc. 4/1, v/v): GC/MSD retention time (min) $10.11,(\mathrm{~m} / \mathrm{z}) 51,57.77 .89$. 115, 128, 140,158, $169(100), 186,201,226.257(\mathrm{M}):{ }^{1} \mathrm{H}$ NMR $\left(300 \mathrm{MHz}, \mathrm{CDCl}_{\hat{3}}\right) \delta 0.88(\mathrm{t}, J=7.2 \mathrm{~Hz}, 3 \mathrm{H}) .1 .32-$ $1.41(\mathrm{~m} .4 \mathrm{H}) \cdot 2.06-2.1+(\mathrm{m} .2 \mathrm{H}) .3 .76(\mathrm{~s} .3 \mathrm{H}) .6 .29(\mathrm{~d} . J=$ $15.5 \mathrm{~Hz}, \mathrm{IH}) .6 .98(\mathrm{~d}, J=15.5 \mathrm{~Hz}, \mathrm{lH}) .7 .35-7.47(\mathrm{~m}, 5 \mathrm{H})$ : ${ }^{13} \mathrm{C} \mathrm{NMR}\left(75 \mathrm{MHz}, \mathrm{CDCl}_{3}\right) \delta 14.1 .22 .8,27.7 .39 .7,49.9$. $52.3,120.1,122.4,126.5,128.8,129.6,137.4,146.3,166.3$ : $(R)-(+)-9(90 \%$ ee $)[\alpha]_{\mathrm{D}}^{2+}+8.0(c 0.50 . \mathrm{MeOH})$.

$(R)$-(-)-4-Cyano-4-phenyloctanoic acid methyl ester $((R)-$ $(-)-10)$. To a solution of $(R)-9(0.13 \mathrm{~g} .0 .51 \mathrm{mmol} .90 \%$ ee $)$ in methanol $(8 \mathrm{~mL})$ was added $10 \% \mathrm{Pd} / \mathrm{C}(10 \mathrm{mg})$ and charged with hydrogen by hỵdrogen-contained balloon. After Ih the reaction mixture was filtered by Celite 545 and the filtrate was concentrated. The crude was purified by column chromatography ( $n$-hexane/EtOAc. 10/1,v/v) to give $(R)-\mathbf{1 0}$.

Yield $(0.13 \mathrm{~g}) 95 \%$ : Rf 0.57 (n-hexane/EtOAc. 5/1, v/v): GC/MSD retention time (min) $9.98,(\mathrm{~m} / \mathrm{z}) 55.59,77.91$. 115,129 (100). 142. 158, 171. 185, 203. 216. 228, 244. 259 $\left(\mathrm{M}^{+}\right):{ }^{l} \mathrm{H}$ NMR $\left(300 \mathrm{MHz}, \mathrm{CDCl}_{3}\right) \delta 0.83(\mathrm{t} . J=7.2 \mathrm{~Hz}, 3 \mathrm{H})$. $1.02-1.11(\mathrm{~m} . \mathrm{lH}) .1 .15-1.29(\mathrm{~m} .2 \mathrm{H}) .1 .30-1.45(\mathrm{~m} . \mathrm{HH})$. $1.81-1.96$ (m. 2H). 2.00-2.11 (m. IH). $2.21-2.35$ (m. 2H). $2.38-2.47(\mathrm{~m}, 1 \mathrm{H}) .3 .59(\mathrm{~s}, 3 \mathrm{H}) .7 .28-7.42(\mathrm{~m}, 5 \mathrm{H}):{ }^{13} \mathrm{C}$ NMR (75 MHz. $\left.\mathrm{CDCl}_{3}\right) \delta 1+.1,22.8,27.7,30.5,36.1,41.2$. $47.9 .52 .1,122.2,126.4 .128 .3,129.4,137.8 .173 .1:(R)-(-)-$ $10(90 \%$ ee $)[\alpha]_{\mathrm{D}}^{3+4}-17.3(c 1.0 . \mathrm{MeOH})$.

(R)-(-)-4-Cyano-4-phenyl-1-octanol $((R)-(-)-3)$. To a solution of $(R)-10(0.13 \mathrm{~g} .0 .48 \mathrm{mmol} .90 \%$ ee $)$ in methanol ( $8 \mathrm{~mL}$ ) was added $\mathrm{NaBH}_{4}(0.18 \mathrm{~g} .4 .8 \mathrm{mmol})$ and refluxed for $5 \mathrm{~h}$. The reaction mixture was quenched by $1 \mathrm{~N}$ aqueous $\mathrm{HCl}$ solution in ice bath and extracted by diethỵl ether $(2 \times 10 \mathrm{~mL})$. The organic layer was purified by column chromatography ( $n$-hexane/EtOAc. $4 / 1$, v/v) to give $(R)-3$. $(R)-(-)-3(90 \%$ ee $)[\alpha]_{\mathrm{D}}^{3 i}-6.29(c 0.35, \mathrm{MeOH})$.

Syntheses of compounds for the determination of absolute configuration of the resolved alcohol $(S)-4$

(S)-2-(4-Chlorophenyl)-2-formylhexanenitrile ((S)-12). (S)-12 was synthesized by the same method as (S)-8 using compound $11(0.15 \mathrm{~g} .0 .63 \mathrm{mmol} .95 \%$ ee) and PCC ( $0.27 \mathrm{~g}$. $1.27 \mathrm{mmol})$

Yield $(0.13 \mathrm{~g}) 87 \%: \mathrm{R}_{\mathrm{f}} 0.26$ (n-hexane/ EtOAc. 4/1, v/v): ${ }^{l} \mathrm{H} \mathrm{NMR}\left(300 \mathrm{MHz}, \mathrm{CDCl}_{3}\right) \delta 0.90(\mathrm{t} . J=7.3 \mathrm{~Hz} .3 \mathrm{H}) .1 .17-$ $1.46(\mathrm{~m} .+\mathrm{H}) .1 .98-2.15(\mathrm{~m}, \mathrm{lH}) .2 .17-2.30(\mathrm{~m} .1 \mathrm{H}) .7 .35-$ $7.47(\mathrm{~m} .5 \mathrm{H}) .9 .42$ (s. $1 \mathrm{H})$.

(S)-t-(t-Chlorophenyl)-t-cyanooct-2-enoic acid methyl ester $((S)-13)$. (S)-13 was sytnthesized by the same method as (R)-9 using (S)-12 (70 $\mathrm{mg} .0 .3 \mathrm{mmol})$ and methyl (triphenylphosphoranylidene)acetate $(0.11 \mathrm{~g} .0 .34 \mathrm{mmol})$.

Yield (72 mg) 83\%: $\mathrm{R}_{\mathrm{f}} 0.46$ (n-hexane/EtOAc. $\left.4 / \mathrm{l}, \mathrm{v} / \mathrm{v}\right)$ : GC/MSD retention time (min) 11.51. (m/2) 57.65,75,88, 101, 113, 128, 140, 149. 163, 174. 185, 193. 203 (100). 212. 220. 235. 248. 260. 276. 29l $\left(\mathrm{M}^{+}\right)$: ${ }^{\mathrm{l}} \mathrm{H}$ NMR $(300 \mathrm{MHz}$. $\left.\mathrm{CDCl}_{3}\right) \delta 0.89(\mathrm{t} . J=7.0 \mathrm{~Hz}, 3 \mathrm{H}), 1.28-1.39(\mathrm{~m}, 4 \mathrm{H}) .2 .02-$ $2.08(\mathrm{~m}, 2 \mathrm{H}), 3.76(\mathrm{~s} .3 \mathrm{H}), 6.27(\mathrm{~d}, J=15.5 \mathrm{~Hz}, \mathrm{lH}) .6 .91(\mathrm{~d}$. $J=15.5 \mathrm{~Hz}, \mathrm{lH}) .7 .25-7.38(\mathrm{~m}, 4 \mathrm{H}):{ }^{13} \mathrm{C} \mathrm{NMR}(75 \mathrm{MHz}$. $\left.\mathrm{CDCl}_{3}\right) \delta 14.1,22.7,27.7,39.7,49.4,52.4,119.7,122.8$. 128.0. 129.7. 134.9.136.0. 145.7. 166.1.

(S)-4-Cyano-4-(4-chlomphenyl)octan-1-ol ((S)-4). (S)-4 was synthesized by the same method as $(R)-3$ using $(S)-14$ (54 mg. $0.18 \mathrm{mmol})$ and $\mathrm{NaBH}_{4}(70 \mathrm{mg} .1 .80 \mathrm{mmol})$.

Yield (35 mg) 73\%: HPLC analysis (Chiralcel OD, $n$ hexane/IPA. 95/5, v/v). retention time (min), $39.58(S)$ and 44. $18(R)$.

Syntheses of compounds for the determination of absolute configuration of the resolved alcohol (S)-6

(S)-2-(3-Oxopropyl)-2-phenylhexanenitrile ((S)-15). (S)-15 was synthesized by the same method as (S)-8 using (S)-3 (0.11 g. $0.46 \mathrm{mmol} .73 \%$ ee) and PCC (0.15 g. 0.69 inmol).

Yield (83 mg) 79\%: $\mathrm{R}_{\mathrm{i}} 0.26$ (n-hexane/EtOAc. 4/1. v/v): ${ }^{\mathrm{l}} \mathrm{H}$ NMR $\left(300 \mathrm{MHz}, \mathrm{CDCl}_{2}\right) \delta 0.86(\mathrm{t}, J=7.2 \mathrm{~Hz}, 3 \mathrm{H}), 1.03-$ $1.19(\mathrm{~m}, \mathrm{lH}) .1 .25-1.50(\mathrm{~m}, 3 \mathrm{H}), 1.88-2.10(\mathrm{~m} .3 \mathrm{H}) .2 .25-$ $2.43(\mathrm{~m}, 2 \mathrm{H}) .2 .63-2.77(\mathrm{~m}, \mathrm{lH}) .7 .32-7.44(\mathrm{~m} .5 \mathrm{H}) .9 .68(\mathrm{~s}$. $\mathrm{IH}):{ }^{13} \mathrm{C}$ NMR $\left(75 \mathrm{MHz}, \mathrm{CDCl}_{3}\right) \delta 14.1,22.8,27.8,33.2$, $40.5,41.3,47.8,122.2,126.2,128.4,129.5,137.8,200.5$.

cis- and trans-(S)-2-Butyl-6-methoxy-2-phenylhex-5enenitrile ( $(S)-16)$. To a solution of (methosymethyl)triphenylphosphonium chloride $(0.15 \mathrm{~g} .0 .43 \mathrm{mmol})$ in THF ( $10 \mathrm{~mL}$ ) was added dropwise $n$-butyl lithium $(0.27 \mathrm{~mL} .0 .43$ mmol. $1.6 \mathrm{M}$ in $n$-hexane) at $78^{\circ} \mathrm{C}$ and stirred for $10 \mathrm{~min}$. ( $\$$ )-15 (83 $\mathrm{mg} .0 .36 \mathrm{mmol}$ ) was added to reaction mixture dropwise at $78^{\circ} \mathrm{C}$ at $30 \mathrm{~min}$ and the mixture was stirred for $10 \mathrm{~h}$ at $\mathrm{rt}$. After the reaction mixture was quenched by ice water $(5 \mathrm{~mL})$ and extracted by diethyl ether $(20 \mathrm{~mL})$. The organic layer was concentrated and purified by column chromatography ( $n$-hexane/EtOAc. $10 / 1, v / v)$ to give $(S)-16$.

Yield (72 mg) 77\%: $\mathrm{R}_{i} 0.56$ (n-hexane/EtOAc. 4/1. v/v): GC/MSD retention time (min) 9.95 and 10.29 . (m/z) 55.63 . $71(100), 78,85,92,103,117,129,136,143,154,166,173$. $186,193,201,215,222,228,242,250,257\left(\mathrm{M}^{+}\right)$.

$(S)$-(+)-2-Butyl-6-methoxy-2-phenylhexanenitrile ((S)$(+)-17)$. ( $S)-17$ was sytnthesized by the same method as $(R)-9$ using (S)-16 (72 mg. $0.28 \mathrm{mmol}$ ).

Yield (65 mg) 90\%: $\mathrm{R}_{\mathrm{i}} 0.42$ (n-hexane/EtOAc. 4/1. v/v): GC/MSD retention time (min) 10.02. (m/2) 55.65.77.87 (100), 103, 116, 129, 143, 158, 173, 186, 203,216,232,244. $259\left(\mathrm{M}^{+}\right):{ }^{1} \mathrm{H}$ NMR $\left(300 \mathrm{MHz}, \mathrm{CDCl}_{3}\right) \delta 0.85(\mathrm{t} . J=7.2 \mathrm{~Hz}$. $3 \mathrm{H}) .1 .00-1.17(\mathrm{~m} . \mathrm{IH}), 1.20-1.34(\mathrm{~m}, 2 \mathrm{H}) .1 .38-1.60(\mathrm{~m}$. $4 \mathrm{H}) .1 .82-2.03(\mathrm{~m}, 4 \mathrm{H}) .3 .28(\mathrm{~s}, 3 \mathrm{H}), 3.29-3.4 \mathrm{l}(\mathrm{m}, 2 \mathrm{H})$. 7.29-7.40 (m. 5H): ${ }^{13} \mathrm{C}$ NMR (75 MHz. $\left.\mathrm{CDCl}_{3}\right) \delta 14.1 .22 .5$. 22.9. 27.7, 29.8. 41.2. 48.6, 58.9, 72.7. 122.9. 126.2, 127.9 . 129.2. 139.0: HPLC analysis (Chiralcel OJ, n-hexane/IPA. 97/3, v/v). retention time (min). $10.78(S)$ and $15.87(R):(S)-$ $(+)-17(74 \%$ ee $)[\alpha]_{\mathrm{D}}^{23}+4.56(\mathrm{c} 0.36 \mathrm{MeOH})$. 


\section{Results and Discussion}

The racemic alcohols (1-6) were obtained in high yield from the reaction of nitriles $\mathbf{1} \mathbf{a}-\mathbf{6} \mathbf{a}$ by successively reacting with [H]'protected alkyl bromide and alkyl bromide. The IHP-protected ether was cleaved by I $\mathrm{N}$ methanolic $\mathrm{HCl}$ to release racemic alcohols (1-6) as shown in Scheme I.

Various lipases from PFL, LAK, CRL and PCL were screened for resolving the racemic alcohols (1-6) by enantioselective transesterification using vinyl acetate as an acyl donor in organic solvents. Results of enzymatic reaction of the alcohols (1-6) with different lipases are summarized in Table 1.

In the transesterification of substrate alcohol $(+)-1$, using PFL lipase, $(R)-1$ was the faster reacting enantiomer, and thus $(S)-1$ remained unreacted. However, the resolved $(R)-1$ showed low ee value. The enzymatic reaction of $(+)-2$ by LAK lipase in the presence of pyridine as additive gave an excellent enantioselectivity in $99 \%$ ee over $34 \%$ conversion, but that of $( \pm)-2$ by LAK lipase in the absence of additive did not give good enantioselectivity. Addition of additives in a lipase-catalyzed reaction is known to significantly enhance both enantioselectivity and reaction efficiency. In the enzymatic reaction of racemic alcohol 2 , the addition of pyridine or 18-crown-6 as additives enhanced enantioselectivity surprisingly. In the enzymatic reaction of (+)-3, $(S)-3$ was the faster reacting enantiomer, yielding $(S)-3 d$ in moderate ee, and leaving $(R)-3$ as an enantiomerically enriched unreacted enantiomer. Also the enzymatic reaction of (-)-4 gave excellent enantiometrically enriched unreacted enantiomer (S)-4.

In the kinetic resolution of substrate alcohol $(+)-\mathbf{5}$, using LAK lipase, $(S)-\mathbf{5}$ was the faster reacting enantiomer. It gave unreacted enantiomer $(R)-5$ in $26 \%$ ee for a reaction time of $1.5 \mathrm{~h}$, and it gave the unreacted enantiomer $(R)-5$ in $99 \%$ ee for a reaction time of $8 \mathrm{~h}$. The enzymatic reaction of $(+)-6$ by

$$
\begin{aligned}
& \text { i } \\
& 1 \mathrm{a} \sim 6 \mathrm{a} \quad 1 \mathrm{~b} \sim 6 \mathrm{~b} \quad 1 \mathrm{c} \sim 6 \mathrm{c}
\end{aligned}
$$

a) NaH. Br(Cl$\left.{ }_{2}\right)_{11} \mathrm{OTISP}$, b) Nali, R-Br, c) $1 \mathrm{~N}$ methanolic $\mathrm{HCl}$

Scheme 1. Syntheses of the racemic alcohols (1-6).<smiles>[X]c1ccc(C([R])(C#N)C([R])O)cc1</smiles>

racemic alcohol (1 6)

\begin{tabular}{|c|c|c|c|c|c|c|c|c|}
\hline \multirow{2}{*}{$\begin{array}{l}\text { Substrate } \\
\text { alcohol }\end{array}$} & \multirow{2}{*}{$\begin{array}{c}\text { I ipase } \\
\text { (mass equiz.) }\end{array}$} & \multirow{2}{*}{ Additives } & \multirow{2}{*}{$\begin{array}{l}\text { Time } \\
\text { (h) }\end{array}$} & \multirow{2}{*}{ Solvent } & \multirow{2}{*}{$\begin{array}{l}\text { Conv: } \\
(\%)^{4}\end{array}$} & \multicolumn{2}{|c|}{$\mathrm{ee}(\%)^{6}$} & \multirow{2}{*}{$\mathrm{E}^{\prime \prime}$} \\
\hline & & & & & & Reacted acelate & Residue alcohol & \\
\hline 1 & PFL(0.1) & - & 1 & n-hexant: [tOAc (9:]) & 52 & $40(R)$ & $44(5)$ & 3.5 \\
\hline 2 & $\mathrm{~L} \wedge \mathrm{K}^{\prime}(0.5)$ & - & 2 & n-hexanc: [tOAc $(9 ; 1)$ & 50 & $76(S)$ & $76(R)$ & 16 \\
\hline 2 & $\mathrm{~L} \wedge \mathrm{k}(0.5)$ & pyridine & 3 & n-hexanc [toAc (9i]) & 34 & $99(5)$ & $52(R)$ & 335 \\
\hline 2 & $\mathrm{~L} \wedge \mathrm{K}(0.5)$ & 18 -crown-6 & 1.5 & n-hexane [toAc (9:1) & 50 & $88(5)$ & $89(R)$ & 47 \\
\hline 3 & PFL(0.1) & - & 0.5 & $n$-hexane & 57 & $73(5)$ & $97(R)$ & 26 \\
\hline 4 & PFL(0.1) & - & 1.5 & $n$-hexane & 74 & $34(R)$ & $99(5)$ & 8.8 \\
\hline 5 & $\mathrm{~L} \wedge \mathrm{K}(0.5)$ & - & 2 & $n$-hexane & 34 & $50(S)$ & $26(R)$ & 3.8 \\
\hline 5 & $\mathrm{~L} \wedge \mathrm{K}(0.5)$ & - & 6 & n-hexane & 61 & $42(S)$ & $66(R)$ & 4.6 \\
\hline 5 & $\mathrm{~L} \wedge \mathrm{K}^{\prime}(0.5)$ & & 8 & n-hexane & 75 & $33(S)$ & $99(R)$ & 8.6 \\
\hline 6 & $\operatorname{CRL}(1)$ & - & 1.5 & $n$-hexane & 40 & $30(S)$ & $20(k)$ & 2.2 \\
\hline 6 & $\operatorname{PCL}(0.5)$ & - & 1.5 & $n$-hexane & 60 & $28(S)$ & $42(R)$ & 2.6 \\
\hline
\end{tabular}

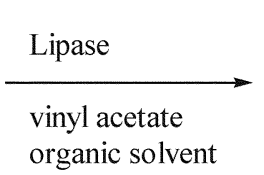

organic solvent

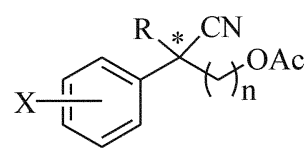

1d $\sim 6 d$

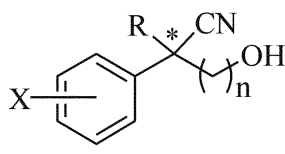

Scheme 2. Lipase-catalyzed reaction of the racemic alcohols (1-6).

Table 1. Results of lipase-catalyzed transesterification of racemic primary alcohols (1-6) using vinul acetate as an acy] donor

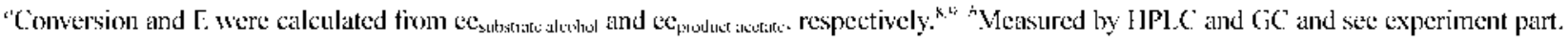


enzymes did not give good enantioselectivity.

The absolute configuration of the resolved alcohol $(R)-1$ was determined by the reaction of the resolved alcohol $(R)-1$ with Jones reagent, followed by treatment with concentrated sulphuric acid to give $(R)$-(-)-3-ethyl-3-(4-nitrophenyl)piperidine-2.6-dione $\left([\alpha]_{\mathrm{D}}^{20}+55.4\right.$ (c $0.2, \mathrm{MeOH}, 48 \%$ ee, lit. $\left.{ }^{10}[\alpha]_{\mathrm{D}}^{20}+129(\mathrm{c} 1.0, \mathrm{MeOH})\right)$ as reported in the literature. (R)-2-Cyano-2-phenyl-1-hexanol $((R)-7)^{6}$ was reacted with pyridinium chlorochromate $(\mathrm{PCC})$ to give $(R)-\mathbf{8}$, which on Wittig olefination with methyl (triphenylphosphoranyl- idene)acetate gave $(R)-9$. $(R)-9$ was reduced by $10 \%$ palladium on charcoal, followed by sodium borohydride to give $(R)$-3. The optical rotation of the resolved alcohol $(R)-3$ was favorably compared with that of the chiral compound 3 synthesized as shown in Scheme 3 .

The absolute configuration of the resolved alcohol $(S)-4$ was determined by the chemical transformation of $(S)-2-(4-$ chlorophenyl)-2-cyano-2-phenyl-1-hexanol $((S)-11)^{6.11}$ to (S)-4 as shown in Scheme 4.

Also the absolute configuration of the resolved alcohol<smiles>N#CC(Br)(CO)c1ccccc1</smiles>

$(R)-7$

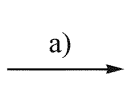<smiles>COC(=O)CC[C@](Br)(C#N)c1ccccc1</smiles>

$(R)-10$<smiles>N#CC(=O)c1ccccc1</smiles>

$(R)-8$

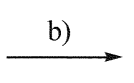

d)<smiles>COC(=O)/C=C/C(Br)(Br)c1ccccc1</smiles>

$(R)-9$

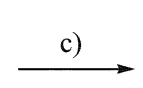

a) $\mathrm{PCC}, \mathrm{CH}_{2} \mathrm{Cl}_{2}, \mathrm{rt}, 86 \%$, b) $\mathrm{Ph}_{3} \mathrm{PCHCO}_{2} \mathrm{CH}_{3}, \mathrm{CH}_{2} \mathrm{Cl}_{2}, 90 \%$

c) $10 \% \mathrm{Pd} / \mathrm{C}, \mathrm{CH}_{3} \mathrm{OH}$, rt, $95 \%$, d) $\mathrm{NaBH}_{4}, \mathrm{CH}_{3} \mathrm{OH}$, reflux, $90 \%$

Scheme 3. Synthesis of authentic $(R)-3$.<smiles>N#C[C@](Br)(CO)c1ccc(Cl)cc1</smiles>

$(S)-11$

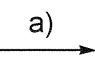<smiles>N#CC(Br)(C=O)c1ccc(Cl)cc1</smiles>

$(S)-12$ b)<smiles>N#C[C@](Br)(CCCO)c1ccccc1</smiles>

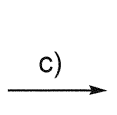<smiles>COC(=O)CC[C@](Br)(C#N)c1ccc(Cl)cc1</smiles>

$(S)-14$

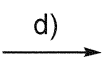<smiles>N#C[C@](Br)(CCCO)c1ccc(Cl)cc1</smiles>

$(S)-4$

a) PCC. CH, $\mathrm{Cl}_{2}, \mathrm{rl}, 87 \%$. b) $\mathrm{Ph}_{3} \mathrm{PCH} \mathrm{CO}_{2} \mathrm{CH}_{3}, \mathrm{Cll}_{2} \mathrm{Cl}_{2} .83 \%$

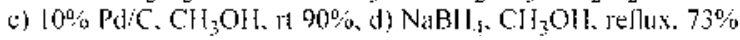

Scheme 4. Synthesis of authentic (S)-4.<smiles>N#C[C@](Br)(CCCO)c1ccccc1</smiles>

$(S)-\mathbf{3}$

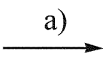<smiles>N#C[C@](Br)(CCC=O)c1ccccc1</smiles>

$(S)-15$

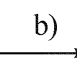<smiles>CO/C=C/CC[C@](Br)(C#N)c1ccccc1</smiles>

$(S)-16$<smiles>N#C[C@](Br)(CCCCO)c1ccccc1</smiles>

The resolved $(S)-6$

\section{$\underset{\mathrm{DMSO}}{\stackrel{\mathrm{CH}_{3} \mathrm{I}, \mathrm{KOH}}{\longrightarrow}}$}

c)

a) $\mathrm{PCC}, \mathrm{CH}_{2} \mathrm{Cl}$, rt. $79 \%$. b) $\mathrm{Ph}_{3} \mathrm{PCHOCH} \mathrm{Cl}_{3} \mathrm{Cl}, n-\mathrm{BuLi},-78^{\circ} \mathrm{C}$ to rt. THF, $77 \%$.

c) $10 \% \mathrm{Pd} / \mathrm{C} \cdot \mathrm{ClI}_{5} \mathrm{OH}, \mathrm{rl}, 90 \%$

Scheme 5. Synthesis of authentic (S)-6. 
(S)-6 was determined by the chemical transformation of $(S)$ 3 to $(S)-17$ as shown in Scheme 5. The resolved alcohol $(S)$ 6 reacted with methyl iodide to give $(S)-17$ and the optical value of $(S)-17$ was compared with that of $(S)-17$ synthesized.

In conclusion. the racemic alcohols (1-6) were resolved by enzymes and their absolute configurations were determined. Also the resolved alcohols (1-6) can be further employed to synthesize various derivatives such as bicyclic amidines. lactones. primary amines aldehỵdes. amides carboxỵlic acid and py ridine.

Acknowledgements. Financial support from Ministry of Science and Technology is gratefully acknowledged and we would like to express our gratefil thanks to Amano enzyme. Co. Ltd. Japan.

\section{References}

1. (a) Pogoreve, M.: Faber K. J. Mol Cat B: Enztmatic 2000, 10. 357. (b) Grigg. R.: Mitchell, T. R. B.: Sutthivaivakit. S.
Tetrahedron Lett. 1981. 22. 4107. (c) Caron, S.: Vazquez, E. Wojcik. T. M. J. Am. Chem. Soc. 2000. 122. 712. (d) Zoltewicz. T. A. Top. Curr. Chent 1975. 59.33 .

2. Carnell. A. T.: Hernandez. M. L. E.: Pettmant. A.: Bickley. T. F. Tetrahedron Lett. $2000,+1.6929$

3. Soine. W. H. Hed Res Rer: 1986, 6, 41 .

4. Fadel, A.: Garcia-Argote. S. Tetahedron: Asymmety 1996, 7. 1159.

5. In1. D. S.: Cheong. C. S.: Lee. S. H.: Park. H.: Youn. B. H. Tetrahedhon: Astnmetiv 1999. 10.3759.

6. Im. D. S: Cheong, C. S.: Lee. S. H.: Youn. B. H: Kim, S. C. Tetrahedon 2000.56, 1309 .

7. (a) Weber, H. K.; Stecher. H.: Faber. K. Biotech. Lett. 1995. 17. 803. (b) Khmelnitsky. Y. L.: Welch. S. H. Clark. D. S.; Dordick. J. S. J. Am Chem. Soc. 1994. 116. 2647. (c) Itoh. T.: Takagi. Y.: Murakami. T.: Hyama. Y.: Tsukube. H. J. Okg. Chem. 1996. 61. 2158.

8. Chen. C.-S.: Fujimoto. Y.: Girdaukas, G.: Sih, C. J. Ant Chent. Soc. 1982, 104, 7294.

9. Kroutil. W. Kleewein. A.: Faber, K. Temahedron: Asymmeny 1997. 8. 3251. A simple program to calculate enantiomeric ratio (E) is freely available at http inww-orgc.tu-graz.ac.at.

10. Hartmant1. R. W. Batzl. C. J. Med Chem. 1986. 29. 1362

11. Ruano. J. L. G.; Garcia, M. C.: Castro, A. M. M.: Ramos, J. H. R. Org. Lett. 2012. 4. 55. 